\title{
A Novel Signature Predicts Recurrence Risk In Breast Cancer Patients
}

Tam Vy Le ${ }^{1}$, Van Quang Ta ${ }^{1}$, Dinh-Truong Nguyen ${ }^{1,2, *}$ and Minh Nam Nguyen ${ }^{1, *}$

${ }^{1}$ School of Biotechnology, Tan Tao University, Duc Hoa, Long An, 850000, Viet Nam., ${ }^{2}$ Center for Research \& Technology Transfer, Tan Tao University, Duc Hoa, Long An, 850000, Viet Nam., *Corresponding authors: E-mail: truong.nguyen@ttu.edu.vn ; minhnam1984@gmail.com

ABSTRACT Breast cancer is one of the most leading causes of death in females all over the world. Although, the incidence rate of breast cancer is low, the death proportion is relatively high. For breast cancer patients, the recurrence ratio is rare, yet it still happens. The highest risk of recurrence usually happens at the early time of treatment, and its severeness depends on the diagnosed stage and the size of the tumor. However, the recurrence prognostic of breast cancer still limited due to the poor prediction strength of input data. Therefore, in this study we used four independent data sets $(n=793)$ with the same chipsets (HG-U133A) of breast cancer patients to investigate a 67 gene signature used in further building the prognostic model for breast cancer patients. Our results finally confirmed that a 67 gene set was significantly associated with the recurrence (RFS) $\left(p=1.66 e^{-17}\right)$, and overall survival (OS) $\left(p=9.27 e^{-06}\right)$. Besides, the gene signature also correlated with survival outcomes, such as RFS ( $p=0.00167)$, and distant metastasis-free survival (DMFS) $(p<0.001)$. Remarkably, our classification analysis results reveal that the breast cancer patients who were categorized into the low-risk group tend to have significantly associated with the longer recurrence time in both training and validation data sets. In conclusion, the 67 gene signature should be considered as potential candidates to be used in building up models or integrated into the current treatment regime for breast cancer.

\section{KEYWORDS}

Microarray

Gene signature

Breast cancer

Prognosis

ARTICLE HISTORY

Received: 15.08.2020

Accepted: 15.09 .2020

Online: 01.10 .2020

DOI: https://doi.org/10.53901/acfq4qa880g

\section{INTRODUCTION}

Breast cancer can occur in both males and females, which is related to genetic and heterogeneity [1] ; however, it is more familiar to females [2]. Even though the early diagnosis by biomarker detection and screening is improved, tumor relapse (local relapse or distant relapse) is the main event that causes death in breast cancer patients [3]. The recurrence of patients who had the first treatment of breast cancer is usually high, and the prognosis for this recurrence is still limited [4]. Therefore, it is necessary to identify biomarkers that could predict the relapse time of patients. DNA microarray was suggested to be a potential method that can identify gene signatures in breast cancer $[5,6,7,8,9]$. The gene signature from only a single data set gave a poor strength of prediction for patients in breast cancer. Thus, the difference from the single training data set, this study introduced testing in multiple data sets which can increase the accuracy of the prognostic value of gene signature. In this work, we identified gene signature over the analysis of RNA expression profiles of 793 breast cancer patients from the Gene Expression Omnibus (GEO, http://www.ncbi. nlm.nih.gov/geo/) database. The comparisons between groups of patients who had relapse or no relapse and groups of patients who had normal breast or cancer breast established a 67 gene signature. Finally, the 67 gene signature was defined by a risk scoring method that was highly predictive of relapse outcomes of breast cancer patients.

\section{MATERIALS AND METHODS}

\section{Patients and gene expression profiles}

All clinical information and gene expression data were retrieved from the Gene Expression Omnibus database http://www.ncbi.nlm. nih.gov/geo/ with the HG-U133A chipset. The follow-up infor- 


\section{Tumor vs. normal}

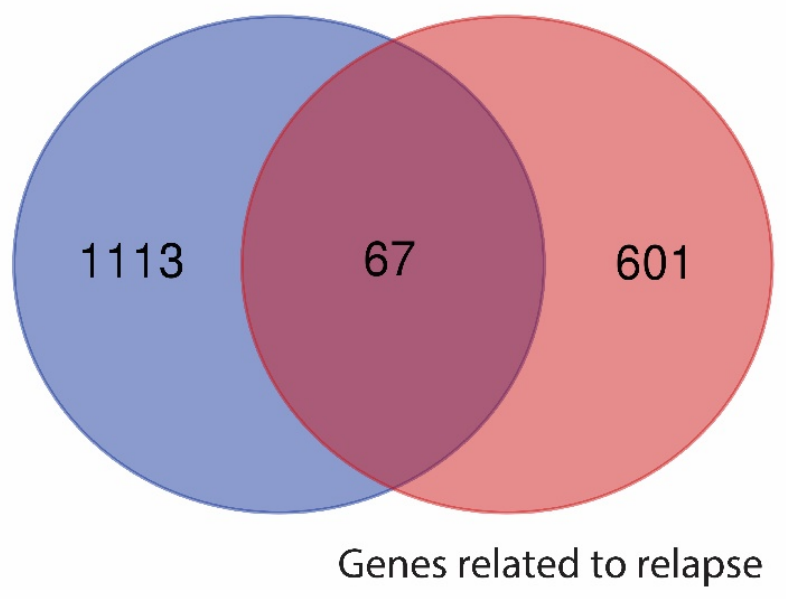

Figure 1 The 67-gene signature in both GSE1456 and GSE15852. 668 gene probes related to a survival of tumor patients from GSE1456, and 1180 gene probes what related to differential expression between tumor and normal patients from GSE15852. After filtering the overlap gene probes, we obtained a 67-gene signature.

mation which included relapse-free survival (RFS), overall survival (OS), and distant metastasis-free survival (DMFS) were selected. Besides, the unfollow-up dataset was excluded from the survival analysis. The gene expression data was normalized from using a robust multiarray averaging method [10,11]. In this analysis, 793 breast cancer patients from four different datasets were used. The GSE1456 ( $n=318$, Karolinska Institute) [12] and GSE15852 ( $n=86$, Institute for Medical Research) [13] were chosen as main databases to find a set of signature genes in relapse patients. Furthermore, GSE11121 $(n=200$, Bayer Technology Services $\mathrm{GmbH}$ ) [14], GSE2990 ( $n=189$, Princess Margaret Cancer Centre) [15] were the validated datasets (Table 1).

\section{Development of the prognostic gene expression signature}

From GSE1456 and GSE15852, 404 breast cancer patients were selected to build a set of signature gene. These genes were developed by BRB-Microarray Tools https://brb.nci.nih.gov/BRB-ArrayTools/ which was an add-in tool in Microsoft Excel. The process was divided into five steps. Firstly, the normalized gene expression from the two datasets was imported into BRB-Array Tools. Secondly, gene probes associated with RFS were identified by the univariate Cox proportional hazard regression $(p<0.001)$ [16] in GSE1456. Thirdly, the gene probes that significantly expressed differently in tumor and normal patients were selected from GSE15852 data set $(p<0.001)$. Forth, the overlapped gene probes from the second and third steps were identified by the Venn diagram. Fifth, the common gene probes from the forth step were applied to the survival risk prediction analysis [17]. In this prediction analysis, a prognostic index was produced for each patient by the formula $\sum_{i} w_{i} x_{i}$ where $\mathrm{w}$ is weight and $\mathrm{x}$ is logged gene expression of the $n^{\text {th }}$ gene, respectively. Patients were classified into high (greater than the median value of prognostic indices) and low (equivalent to or less than the median value of prognostic indices) risk group based on the value of prognostic indices (Fig. 1).

\section{Validation of the prognostic signature}

The validation datasets were from different sources and prediction model was developed using BRB-Array Tools [18]. Complete leave-one-out cross validation (LOOCV) that displayed the accuracy of the prediction was $99 \%$ for the compound covariate predictor (CCP) [19]. Kaplan-Meier Plots were performed the survival analysis of patients in two classified risk groups. Moreover, to evaluate the survival risk of two groups, Chi-square and t-tests were applied.

\section{STRING analysis}

Protein-protein interaction was achieved by a functional protein association network named Search Tool for the Retrieval of Interacting Genes/Proteins (STRING) https://string-db.org/. This included text mining, experiments, databases, co-expression, neighborhood, gene fusion, and co-occurrence criteria [20].

\section{Statistical methods of microarray data}

Data analysis including microarray data and heat map were based on BRB-Microarray Tools. Kaplan-Meier Plot, Chi-square test, a two-sample t-test were performed by the R language environment www.r-project.org and the statistical package for social sciences (SPSS) (version 20, SPSS Inc., Chicago, IL, USA). A p-value less than 0.05 was considered significant.

\section{RESULT}

Identification of the genes related to relapse-free survival and significantly different gene expression between breast cancer and normal tissues

We developed a 67 gene signature which was associated with relapse-free survival from breast cancer patients and significantly different gene expression from breast cancer and normal patients (Fig. 1). In this study, GSE1456 was the training dataset with the relapse clinical outcome considered as the main follow up information. GSE15852 was used as independent data that could increase the significant accuracy of gene signature. After overlapping 668 RFS-associated gene probes in GSE1456 ( $p<0.001)$, and 1180 gene probes that significant different expressed in GSE15852 $(p<0.001)$, 67 gene probes were identified (Table 2 ). In 67 gene set, there were 59 annotated genes, in there, 5 genes that had more than one probe, 4 probes had no annotated genes, and 50 genes had only one probe. A flow chart used to develop a gene set was presented in Fig. 2.

\section{Develop a gene signature to predict a recurrence outcome}

In GSE1456, patients were classified into high $(n=79)$ and low $(n=80)$ risk groups based on the gene expression of 67 gene probes and the recurrence information from each patient. The classification was following the median of prognostic index generated by Principal Component Analysis. These 67 gene set showed distinct clustering of genes in tumor patients (Fig. 3A). In addition, the survival differences of the predicted high risk and low risk groups were performed by Kaplan-Meier curves in RFS $(p=1.66 \exp (-07)$, Fig. 3B), and OS ( $p=9.27 \exp (-06)$, Fig. 3C). The results showed that patients in low group had a longer recurrence and survival time. Taken together, the 67 gene signature was correlated to the recurrence and overall survival outcomes of breast cancer patients.

\section{Validation of the $\mathbf{6 7}$ gene predictive signature}

To evaluate the accuracy of the 67 gene signature, we validated it in two independent data sets of breast cancer. The procedure of 


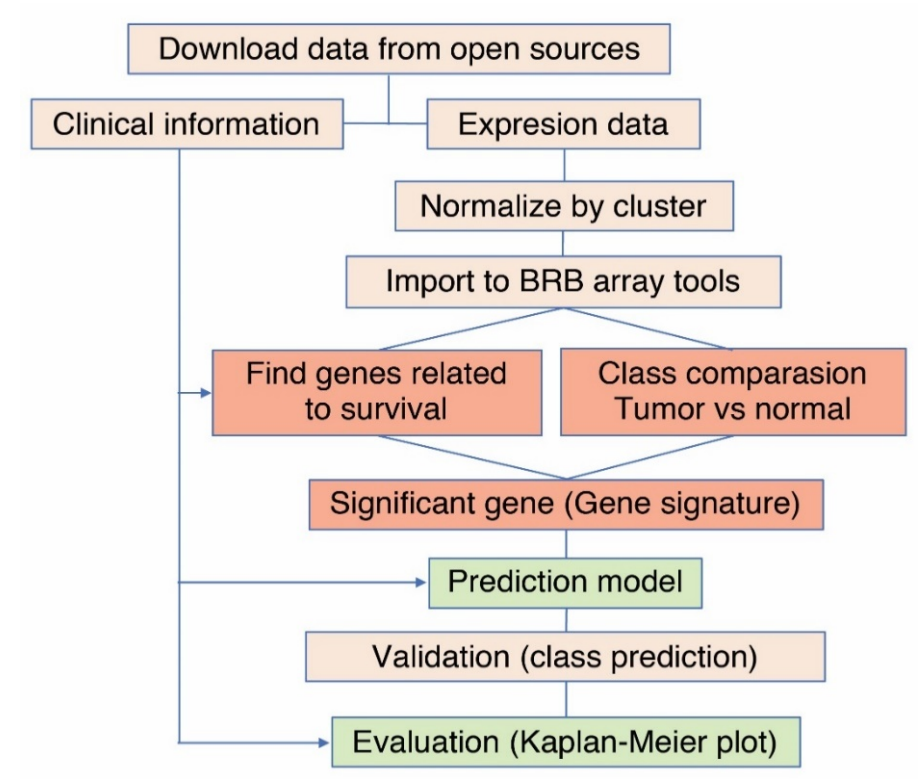

Figure 2 A flow chart of developing the gene signature.

Databases from GSE1456 and GSE15852 were imported into BRBArray Tools. Then, genes related to survival in GSE1456 overlapped with genes differentially expressed between tumor and normal patients in GSE15852. The Prediction model was built based on the 67-gene signature and validated from GSE11121 and GSE2990. The results were visualized by the heat map and Kaplan-Meier Plot.

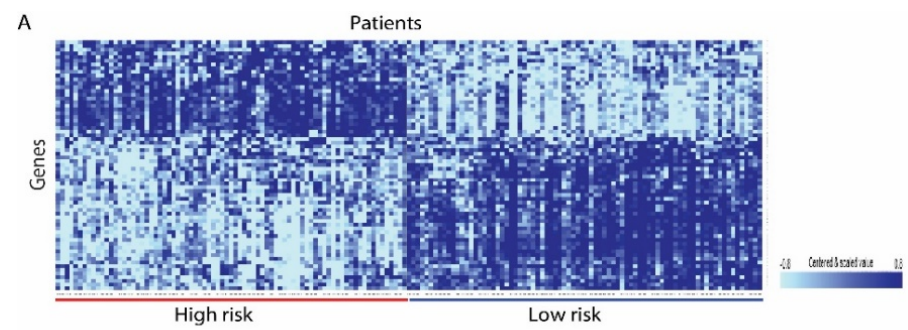

B

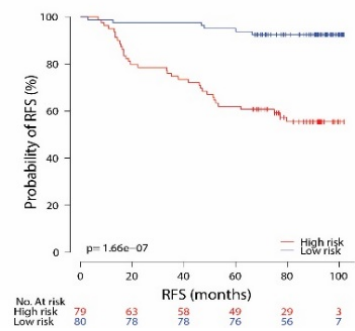

C

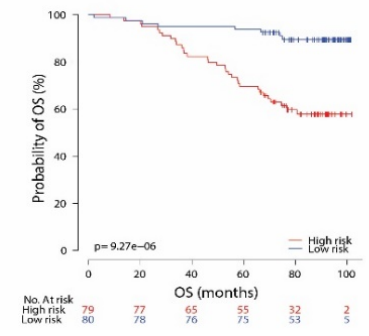

Figure 3 The 67 gene signature in the training dataset

(GSE1456). (A) Heatmap of 67 gene expression of tumor patients. (B) Kaplan-Meier Plot of recurrence survival of patients who had recurrence or recurrence-free status. The high-risk group of patients displayed a shorter recurrence time compared to patients with low risk. (C) Similarity as (B), the high-risk group showed the short-lived time than the low-risk one in tumor patients.

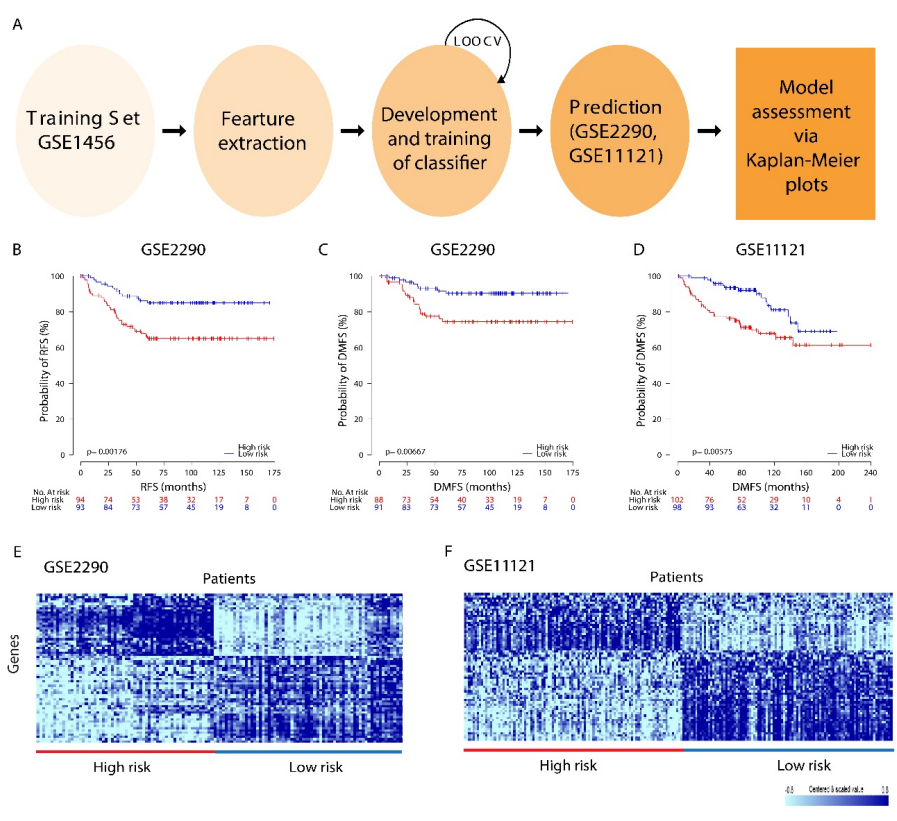

Figure 4 The validation of the 67 gene signature in GSE2290 and GSE11121. (A) A workflow of validation of 67-gene signature in both datasets. Our classification divided patients in GSE2290 into high- and low risk groups with the significant p-value associated with the (B) recurrence and (C) DMFS. (D) The DMFS in GSE11121 also significantly correlated with the predicted groups classified by the 67-gene signature. Besides, the gene signature distributed the patients into two clusters in validation datasets as well as the training dataset $(\mathrm{E}, \mathrm{F})$.

validation was provided in Fig. 4A. In the GSE2290 validation data set, the 67 gene signature predicted patients into high $(n=94)$ and low $(n=93)$ risk groups. Our classification was significant associated with the RFS ( $p=0.00176$, Fig. $4 \mathrm{~B})$, and DMFS ( $p=0.00667$, Fig. 3C). In the GSE11121 validation data set, 102 and 98 patients were classified into high risk and low risk groups, respectively. Accordingly, the DMFS significant correlated to our classification $(p=0.00575$, Fig. 3D). Moreover, the 67 gene signature also categorized the patients into two clusters (Fig. 3E, 3F) in each validation data sets. Altogether, the 67 gene signature was successfully evaluated in both validation data sets.

\section{Protein interaction network of $\mathbf{6 7}$ gene signature}

The protein network of 59 annotated genes of 67 gene signature in breast cancer patients was generated by STRING. There were 41 of 59 genes that had a closed connection in a single network, 18 genes had no connection (Fig. 5).

\section{DISCUSSION}

[] The recurrence of breast cancer after treatment was an unwanted event that every patient has not expected. Many efforts have been made to enhance the clinical management of breast cancer; however, the mortality rate is still rising [21, 22]. Furthermore, a vast quantity of patients had to face breast cancer return within two decades. Due to the heterogeneity in breast cancer, traditional methods (such as tumor size, tumor type, stage) were not enough efficiency to predict the risk of recurrence in tumor patients [21]. The development of a DNA microarray was considered as a potential method of generating gene signatures and used to predict 


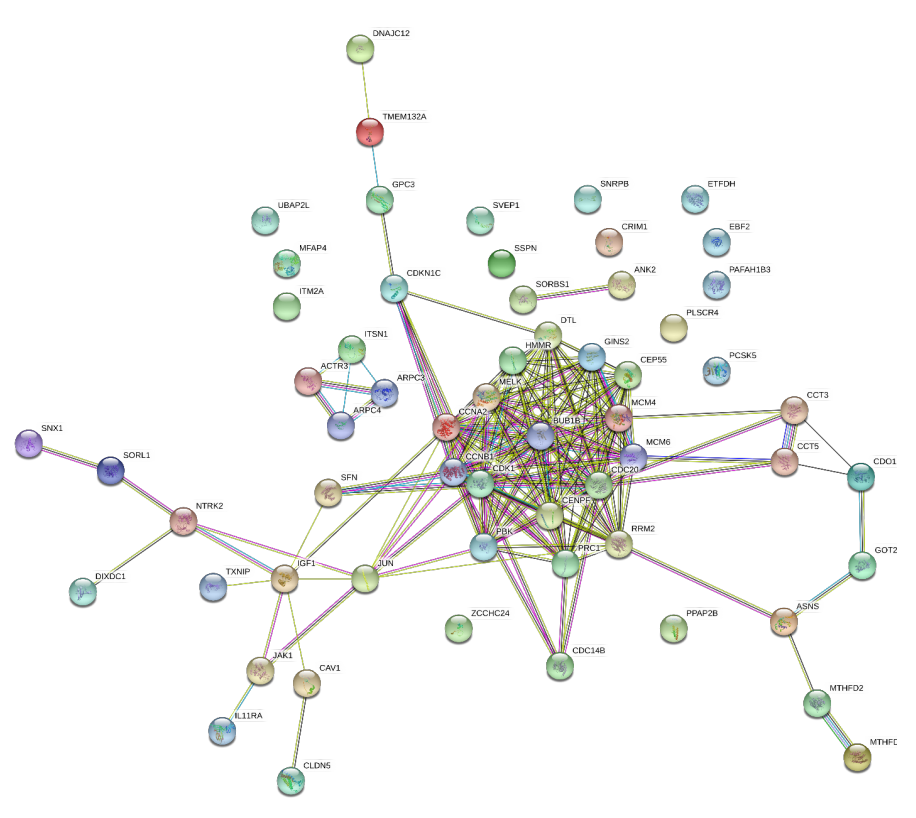

Figure 5 The connection of gene signature generated by STRING. There were 59 annotated genes of 67 gene probes. In the network, only 55 genes had a closed connection with each other.

recurring, survival, and metastasis risks in breast cancer. In this study, we developed a novel gene signature that could accurately predict the recurrence outcome of breast cancer patients. The robustness of the 67 gene signature was supported by the reproducibility through a significant association between the predicted outcome and patient prognosis in two independent validation data sets. The gene signature mainly contains many genes playing critical biology roles in breast cancer progression. Some of these relate to proliferation functions of cells, such as CAV1 [23], CCNA2 [24], CDC20 [25], CDK1 [26], CLDN5 [27], GPC3 [28], IGF1 [29], JUN [30], MELK [31], NTRK2 [32], PRC1 [33], SFN [34], and TXNIP [35]. Besides, some genes are involved in angiogenesis, migration, and inflammation in many cancers, such as CLDN5 [27], JAK1 [36], IGF1 [37], PLPP3 [38], and PBK [39]. Moreover, several genes are related to the Wnt signaling pathway, a hallmark of many cancers, including CAV1 [23], DIXDC1 [40], GPC3 [28], and PLPP3 [38]. Furthermore, the signature also had oxidoreductaserelated genes, such as CDO1 [41], ETFDH [42], MTHFD1 [43], MTHFD2 [44], and RRM2 [45]. Therefore, we argue that the many genes in this signature are involved in proliferation, angiogenesis, migration, and inflammation in breast cancer. In conclusion, we identified the 67 gene signature for prognostic breast cancer to predict recurrence outcomes. The results showed that 67 gene set significantly correlated with survival outcomes, such as recurrence, survival, and metastasis status. In addition, the predictive value and robustness were successfully validated in two independent cohorts of breast cancer patients. The high-risk group had a shorter time of recurrence, and metastasis in both the training, and validation datasets ( $p<0.001$, Fig. 3,4$)$. This study provides a potential tool for the prediction of recurrence in breast cancer in the age of personalized medicine.

\section{COMPETING INTERESTS}

The authors declare that they have no competing interests.

\section{ACKNOWLEDGMENTS}

This research is funded by Tan Tao University Foundation for Science and Technology Development under grant number TTU.RS.19.305.011

\section{REFERENCES}

[1] Laura J. van 't Veer, Hongyue Dai, Marc J. van de Vijver, Yudong D. He, Augustinus A. M. Hart, Mao Mao, Hans L. Peterse, Karin van der Kooy, Matthew J. Marton, Anke T. Witteveen, George J. Schreiber, Ron M. Kerkhoven, Chris Roberts, Peter S. Linsley, René Bernards, and Stephen H. Friend. Gene expression profiling predicts clinical outcome of breast cancer. Nature, 415(6871):530-536, Jan 2002.

[2] Carol E. DeSantis, Jiemin Ma, Mia M. Gaudet, Lisa A. Newman, Kimberly D. Miller, Ann Goding Sauer, Ahmedin Jemal, and Rebecca L. Siegel. Breast cancer statistics, 2019. CA: A Cancer Journal for Clinicians, 69(6):438-451, 2019.

[3] Meng Zhou, Lei Zhong, Wanying Xu, Yifan Sun, Zhaoyue Zhang, Hengqiang Zhao, Lei Yang, and Jie Sun. Discovery of potential prognostic long non-coding rna biomarkers for predicting the risk of tumor recurrence of breast cancer patients. Scientific Reports, 6:31038, 082016.

[4] Abel Matondo, Yong Hwa Jo, Muhammad Shahid, Tae Choi, Nam Nguyen, Ngoc Nguyen, Salima Akter, Insug Kang, Joohun Ha, Chi Maeng, Si-Young Kim, Ju-Seog Lee, Jayoung Kim, and Sung Soo Kim. The prognostic 97 chemoresponse gene signature in ovarian cancer. Scientific Reports, 7, 122017.

[5] Mahsa Marzancola, Abootaleb Sedighi, and Paul Li. DNA Microarray-Based Diagnostics, volume 1368, pages 161-178. 05 2016.

[6] Gabriele Schricker, Rudolf Napieralski, Aurelia Noske, Elodie Piednoir, Olivia Manner, Elisabeth Schüren, Jürgen Lauber, Jonathan Perkins, Viktor Magdolen, Manfred Schmitt, Kurt Ulm, Wilko Weichert, Marion Kiechle, John Martens, and Olaf Wilhelm. Clinical performance of an analytically validated assay in comparison to microarray technology to assess pitx2 dna-methylation in breast cancer. Scientific Reports, 8, 122018.

[7] Marc Vijver, Yudong He, Laura van 't Veer, Hongyue Dai, Augustinus Hart, Dorien Voskuil, George Schreiber, Johannes Peterse, Chris Roberts, Matthew Marton, Mark Parrish, Douwe Atsma, Anke Witteveen, Annuska Glas, Leonie Delahaye, Tony Velde, Harry Bartelink, Sjoerd Rodenhuis, Emiel Rutgers, and Rene Bernards. A gene-expression signature as a predictor of survival in breast cancer. The New England journal of medicine, 347:1999-2009, 122002.

[8] Renaud Sabatier, Pascal Finetti, Nathalie Cervera, Agnes Tallet, Mohamed Benchalal, Gilles Houvenaeghel, Jocelyne Jacquemier, Daniel Birnbaum, and François Bertucci. Gene expression profiling and its utility in prediction of local relapse after breast-conserving therapy in early breast cancer. Cancer genomics proteomics, 8:199-209, 072011.

[9] Rui Liu, Xinhao Wang, Grace Chen, Piero Dalerba, Austin Gurney, Timothy Hoey, Gavin Sherlock, John Lewicki, Kerby Shedden, and Michael Clarke. The prognostic role of a gene signature from tumorigenic breast-cancer cells. The New England journal of medicine, 356:217-26, 022007.

[10] R. Irizarry, B. Hobbs, F. Collin, Y. Beazer-Barclay, Kristen J Antonellis, U. Scherf, and T. Speed. Exploration, normalization, and summaries of high density oligonucleotide array probe level data. Biostatistics, 4 2:249-64, 2003.

[11] Salima Akter, Tae Gyu Choi, Minh Nam Nguyen, Abel Matondo, Jin Hwan Kim, Yong Hwa Jo, Ara Jo, Muhammad 
Shahid, Dae Young Jun, Ji Youn Yoo, Yen Ngoc Nguyen Ngo, Seong Wook Seo, Liaquat Ali, Ju Seog Lee, Kyung Sik Yoon, Wonchae Choe, Insug Kang, Joohun Ha, Jayoung Kim, and Sung Soo Kim. Prognostic value of a 92-probe signature in breast cancer. Oncotarget, 6(17):15662-15680, 2015.

[12] Yudi Pawitan, Judith Bjöhle, Lukas Amler, Anna-Lena Borg, Suzanne Egyhazi Brage, Per Hall, Xia Han, Lars Holmberg, Fei Huang, Sigrid Klaar, Edison Liu, Lance Miller, Hans Nordgren, Alexander Ploner, Kerstin Sandelin, Peter Shaw, Johanna Smeds, Lambert Skoog, Sara Wedrén, and Jonas Bergh. Pawitan $y$, bjöhle j, amler l, borg al, egyhazi s, hall $p$, han $\mathrm{x}$, holmberg 1 , huang $\mathrm{f}$, klaar $\mathrm{s}$, liu et, miller 1 , nordgren $\mathrm{h}$, ploner a, sandelin $\mathrm{k}$, shaw pm, smeds j, skoog l, wedrén s, bergh jgene expression profiling spares early breast cancer patients from adjuvant therapy: derived and validated in two population-based cohorts. breast cancer res 7: R953-r964. Breast cancer research : BCR, 7:R953-64, 022005.

[13] Ivyna Bong, Zubaidah Zakaria, Rohaizak Muhammad, Abdullah Norlia, Naqiyah Ibrahim, Emran Aina, Nor Abdullah, and akmal Sharifah. Gene expression patterns distinguish breast carcinomas from normal breast tissues: The malaysian context. Pathology, research and practice, 206:223-8, 042010.

[14] Marcus Schmidt, Daniel Böhm, Christian Törne, Eric Steiner, Alexander Puhl, Henryk Pilch, Hans-Anton Lehr, Jan Hengstler, Heinz Kölbl, and Mathias Gehrmann. The humoral immune system has a key prognostic impact in node-negative breast cancer. Cancer research, 68:5405-13, 082008.

[15] Christos Sotiriou, Pratyaksha Wirapati, Sherene Loi, Adrian Harris, Steve Fox, Johanna Smeds, Hans Nordgren, Pierre Farmer, Viviane Praz, Benjamin Haibe-Kains, Christine Desmedt, Denis Larsimont, Fatima Cardoso, Hans Peterse, Dimitry Nuyten, Marc Buyse, Marc J. Van de Vijver, Jonas Bergh, Martine Piccart, and Mauro Delorenzi. Gene Expression Profiling in Breast Cancer: Understanding the Molecular Basis of Histologic Grade To Improve Prognosis. JNCI: Journal of the National Cancer Institute, 98(4):262-272, 022006.

[16] T.-K. Jenssen, W. Kuo, T. Stokke, and E. Hovig. Associations between gene expressions in breast cancer and patient survival. Human Genetics, 111(4):411-420, Oct 2002.

[17] Eric Bair and Robert Tibshirani. Semi-supervised methods to predict patient survival from gene expression data. PLoS biology, 2:E108, 052004.

[18] Richard Simon, Amy Lam, Ming-Chung Li, Michael Ngan, Supriya Menenzes, and Yingdong Zhao. Analysis of gene expression data using brb-array tools. Cancer informatics, 3:117, 022007.

[19] Michael Radmacher, Lisa Mcshane, and Richard Simon. A paradigm for class prediction using gene expression profiles. Journal of computational biology : a journal of computational molecular cell biology, 9:505-11, 022002.

[20] Damian Szklarczyk, Andrea Franceschini, Stefan Wyder, Sofia Forslund, Davide Heller, Jaime Huerta-Cepas, Milan Simonovic, Alexander Roth, Alberto Santos, Kalliopi Tsafou, Michael Kuhn, Peer Bork, Lars Jensen, and Christian von Mering. String v10: Protein-protein interaction networks, integrated over the tree of life. Nucleic acids research, 43, 10 2014.

[21] Aamir Ahmad. Breast Cancer Statistics: Recent Trends, pages 1-7. 082019.

[22] R. Edward Hendrick, Jay Baker, and Mark Helvie. Breast cancer deaths averted over 3 decades. Cancer, 125, 022019.

[23] Giusy Fiucci, Dana Ravid, Reuven Reich, and Mordechai Lis- covitch. Caveolin-1 inhibits anchorage-independent growth, anoikis and invasiveness in mcf-7 human breast cancer cells. Oncogene, 21:2365-75, 052002.

[24] Tian Gao, Yong Han, Ling Yu, Sheng Ao, ZiYu li, and Jiafu ji. Ccna2 is a prognostic biomarker for er+ breast cancer and tamoxifen resistance. PloS one, 9:e91771, 032014.

[25] H Karra, H Repo, Ilmari Ahonen, Eliisa Löyttyniemi, Reino Pitkänen, M Lintunen, Teijo Kuopio, Mirva Söderström, and Pauliina Kronqvist. Cdc20 and securin overexpression predict short-term breast cancer survival. British journal of cancer, 110 052014.

[26] Jia-Yi Qian, Jian Gao, Xi Sun, Meng-Da Cao, Liang Shi, TianSong Xia, Wenbin Zhou, Shui Wang, Qiang Ding, and Ji-Fu Wei. Kiaa1429 acts as an oncogenic factor in breast cancer by regulating cdk1 in an n6-methyladenosine-independent manner. Oncogene, 38:1, 072019.

[27] Attila Szász, Zsuzsa Schaff, László Harsányi, István Molnár, Zsolt Baranyai, István Besznyák, Attila Zaránd, Ferenc Salamon, and Janina Kulka. Expression of tight junction molecules in breast carcinomas analysed by array pcr and immunohistochemistry. Pathology oncology research : POR, 18:593-606, 12 2011.

[28] YY Xiang, Virginia Ladeda, and Jorge Filmus. Glypican-3 expression is silenced in human breast cancer. Oncogene, 20:740812, 122001.

[29] Timothy Key, Paul Appleby, Gillian Reeves, and Andrew Roddam. Insulin-like growth factor 1 (igf1), igf binding protein 3 (igfbp3), and breast cancer risk: Pooled individual data analysis of 17 prospective studies. The lancet oncology, 11:530-42, 06 2010.

[30] Marije Vleugel, Astrid Greijer, Reinhard Bos, Elsken Wall, and Paul Diest. c-jun activation is associated with proliferation and angiogenesis in invasive breast cancer. Human pathology, 37:668-74, 072006

[31] Mark Pickard, Andrew Green, Ian Ellis, Carlos Caldas, Vanessa Hedge, Mirna Maarabouni, and Gwyn Williams. Dysregulated expression of fau and melk is associated with poor prognosis in breast cancer. Breast cancer research: $B C R, 11,09$ 2009.

[32] Erin Young, Debra Kelly, Insop Shim, Kyle Baumbauer, Angela Starkweather, and Debra Lyon. Variations in comt and ntrk2 influence symptom burden in women undergoing breast cancer treatment. Biological research for nursing, 19:1099800417692877, 022017.

[33] Arata Shimo, Toshihiko Nishidate, Tomohiko Ohta, Mamoru Fukuda, Yusuke Nakamura, and Toyomasa Katagiri. Elevated expression of protein regulator of cytokinesis 1, involved in the growth of breast cancer cells. Cancer Science, 98(2):174-181, 2007.

[34] Syed Musthapa Meeran, Shweta N. Patel, and Trygve O. Tollefsbol. Sulforaphane causes epigenetic repression of htert expression in human breast cancer cell lines. PLOS ONE, 5, 2010.

[35] Liangliang Shen, John M. O'Shea, Mohan R. Kaadige, Stéphanie Cunha, Blake R. Wilde, Adam L. Cohen, Alana L. Welm, and Donald E. Ayer. Metabolic reprogramming in triple-negative breast cancer through Myc suppression of TXNIP. Proceedings of the National Academy of Sciences, 112(17):5425-5430, April 2015.

[36] Daniel Stover, Carlos Alcazar, Jane Brock, Hao Guo, Beth Overmoyer, Justin Balko, Qiong Xu, Aditya Bardia, Sara Tolaney, Rebecca Gelman, Maxwell Lloyd, Yu Wang, Yaomin 
Xu, Franziska Michor, Vivian Wang, Eric Winer, Kornelia Polyak, and Nancy Lin. Phase ii study of ruxolitinib, a selective jak1/2 inhibitor, in patients with metastatic triplenegative breast cancer. npj Breast Cancer, 4, 122018.

[37] K DeLellis, Sue Ingles, L Kolonel, Roberta McKean-Cowdin, Brian Henderson, Frank Stanczyk, and N Probst-Hensch. Igfi genotype, mean plasma level and breast cancer risk in the hawaii/los angeles multiethnic cohort. British journal of cancer, 88:277-82, 012003.

[38] Hongchang Dong, Shuai Zhang, Yu Wei, Chunyan Liu, Na Wang, Pan Zhang, Jingling Zhu, and Jin Huang. Bioinformatic analysis of differential expression and core genes in breast cancer. International journal of clinical and experimental pathology, 11:1146-1156, 032018.

[39] Chikako Fukukawa, Koji Ueda, Toshihiko Nishidate, Toyomasa Katagiri, and Yusuke Nakamura. Critical roles of lgn/gpsm2 phosphorylation by pbk/topk in cell division of breast cancer cells. Genes, chromosomes cancer, 49:861-72, 10 2010.

[40] Jifu Song, Zhibin Guan, Maojiang Li, Sha Sha, Chao Song, Zhiwei Gao, and Yongli Zhao. Microrna-154 inhibits the growth and invasion of gastric cancer cells by targeting dixdc1/wnt signaling. Oncology Research Featuring Preclinical and Clinical Cancer Therapeutics, 26, 082017.

[41] Dimo Dietrich, Manuel Krispin, Jörn Dietrich, Anne Fassbender, Jörn Lewin, Nadia Harbeck, Manfred Schmitt, Serenella Eppenberger-Castori, Vincent Vuaroqueaux, Frederique Spyratos, John Foekens, Ralf Lesche, and John Martens. Cdo1 promoter methylation is a biomarker for outcome prediction of anthracycline treated, estrogen receptor-positive, lymph node-positive breast cancer patients. BMC cancer, 10:247, 062010.

[42] Yaxun Wu, Xingsong Zhang, Rong Shen, Jieyu Huang, Xiaoyun Lu, Guihua Zheng, and Xudong Chen. Expression and significance of etfdh in hepatocellular carcinoma. Pathology Research and Practice, 215:152702, 102019.

[43] Maja Krajinovic. Mthfd1 gene: Role in disease susceptibility and pharmacogenetics. Pharmacogenomics, 9:829-32, 082008.

[44] Feng Liu, Yang Liu, Chuan He, Li Tao, Xiaoguang He, Hongtao Song, and Guoqiang Zhang. Increased mthfd2 expression is associated with poor prognosis in breast cancer. Tumour biology : the journal of the International Society for Oncodevelopmental Biology and Medicine, 35:8685-90, 052014.

[45] Wen-Hui Liang, Na Li, Zhi-Qing Yuan, Xin-Lai Qian, and Zhi-Hui Wang. Dscam-as1 promotes tumor growth of breast cancer by reducing mir-204-5p and up-regulating rrm2: The association of dscam-as1 with mir-204-5p and rrm2 in bc. Molecular Carcinogenesis, 58:461-473, 112018.

\section{APPENDIX}

Table 2 The first 67 genes are significant at the nominal 0.99

level of the univariate test

\begin{tabular}{llllll}
\hline No. & FDR & Probe Set & Symbol & Name & Entrez ID \\
\hline 1 & 0.00011 & 209220_at & GPC3 & Glypican 3 & 2719 \\
2 & 0.00021 & 208022_s_at & CDC14B & Cell division cycle 14B & 8555 \\
3 & 0.00026 & 201377_at & UBAP2L & Ubiquitin associated protein 2 like & 9898 \\
4 & 0.0384 & 202712_s_at & & & \\
\hline
\end{tabular}


Table 2 The first 67 genes are significant at the nominal 0.99 level of the univariate test

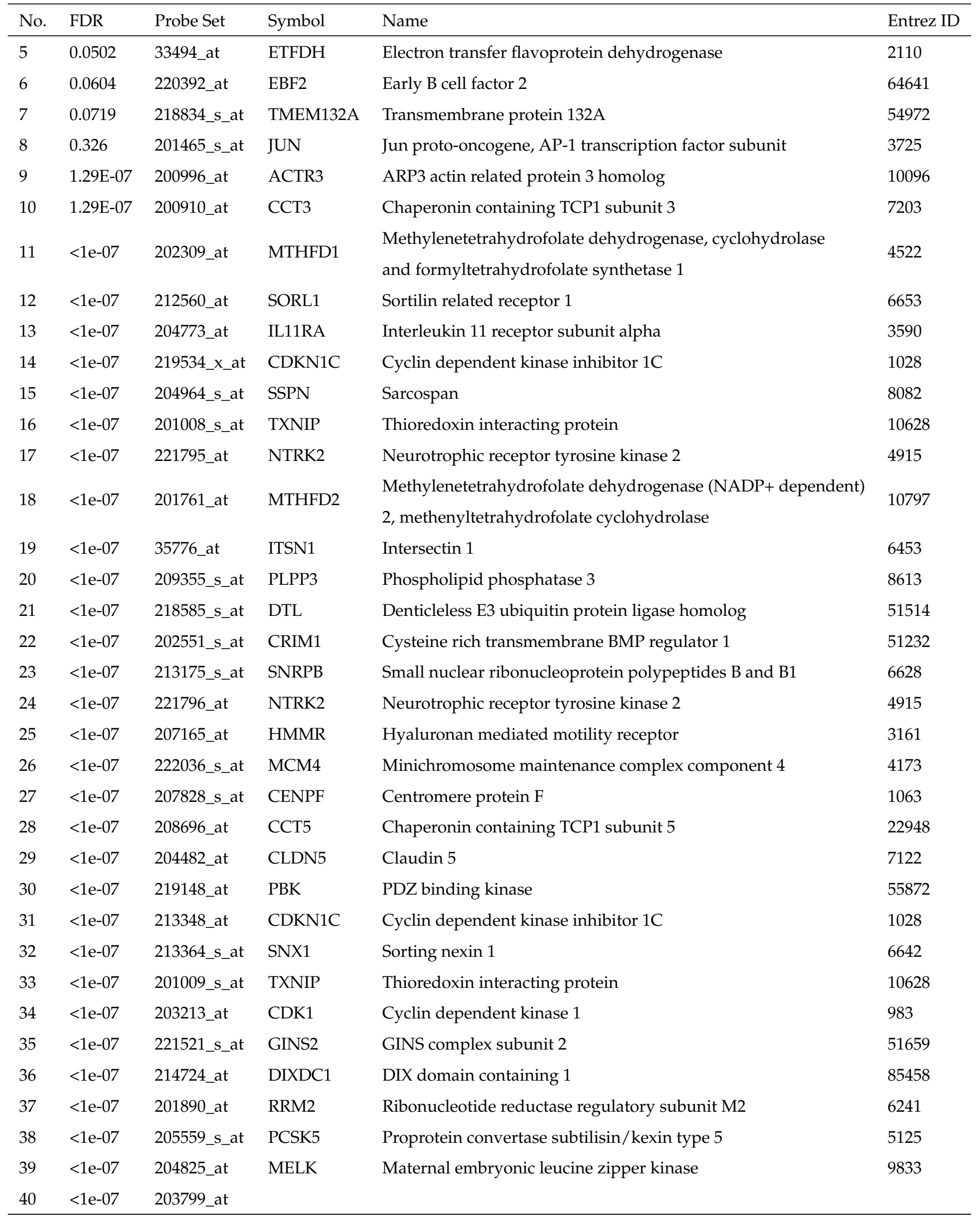


Table 2 The first 67 genes are significant at the nominal 0.99 level of the univariate test

\begin{tabular}{|c|c|c|c|c|c|}
\hline No. & FDR & Probe Set & Symbol & Name & Entrez ID \\
\hline 41 & $<1 \mathrm{e}-07$ & 213226_at & CCNA2 & Cyclin A2 & 890 \\
\hline 42 & $<1 \mathrm{e}-07$ & 219563_at & & & \\
\hline 43 & $<1 \mathrm{e}-07$ & 213247_at & SVEP1 & $\begin{array}{l}\text { Sushi, von Willebrand factor type A, EGF } \\
\text { and pentraxin domain containing } 1\end{array}$ & 79987 \\
\hline 44 & $<1 \mathrm{e}-07$ & 218542_at & CEP55 & Centrosomal protein 55 & 55165 \\
\hline 45 & $<1 \mathrm{e}-07$ & 202920_at & ANK2 & Ankyrin 2 & 287 \\
\hline 46 & $<1 \mathrm{e}-07$ & 202746_at & ITM2A & Integral membrane protein $2 \mathrm{~A}$ & 9452 \\
\hline 47 & $<1 \mathrm{e}-07$ & 211577_s_at & IGF1 & Insulin like growth factor 1 & 3479 \\
\hline 48 & $<1 \mathrm{e}-07$ & 218009_s_at & PRC1 & Protein regulator of cytokinesis 1 & 9055 \\
\hline 49 & $<1 \mathrm{e}-07$ & 209894_at & & & \\
\hline 50 & $<1 \mathrm{e}-07$ & 204154_at & CDO1 & Cysteine dioxygenase type 1 & 1036 \\
\hline 51 & $<1 \mathrm{e}-07$ & 202870_s_at & $\mathrm{CDC} 20$ & Cell division cycle 20 & 991 \\
\hline 52 & $<1 \mathrm{e}-07$ & 218087_s_at & SORBS1 & Sorbin and $\mathrm{SH} 3$ domain containing 1 & 10580 \\
\hline 53 & $<1 \mathrm{e}-07$ & 212713_at & MFAP4 & Microfibril associated protein 4 & 4239 \\
\hline 54 & $<1 \mathrm{e}-07$ & 218901_at & PLSCR4 & Phospholipid scramblase 4 & 57088 \\
\hline 55 & $<1 \mathrm{e}-07$ & 212423_at & $\mathrm{ZCCHC} 24$ & Zinc finger CCHC-type containing 24 & 219654 \\
\hline 56 & $<1 \mathrm{e}-07$ & 212097_at & CAV1 & Caveolin 1 & 857 \\
\hline 57 & $<1 \mathrm{e}-07$ & 209542_x_at & IGF1 & Insulin like growth factor 1 & 3479 \\
\hline 58 & $<1 \mathrm{e}-07$ & 212419_at & $\mathrm{ZCCHC} 24$ & Zinc finger CCHC-type containing 24 & 219654 \\
\hline 59 & $<1 \mathrm{e}-07$ & 209540_at & IGF1 & Insulin like growth factor 1 & 3479 \\
\hline 60 & $<1 \mathrm{e}-07$ & 209541_at & IGF1 & Insulin like growth factor 1 & 3479 \\
\hline 61 & $2.48 \mathrm{E}-07$ & 216894_x_at & CDKN1C & Cyclin dependent kinase inhibitor $1 \mathrm{C}$ & 1028 \\
\hline 62 & $2.48 \mathrm{E}-07$ & 201648_at & JAK1 & Janus kinase 1 & 3716 \\
\hline 63 & 3.65E-07 & 205047_s_at & ASNS & Asparagine synthetase (glutamine-hydrolyzing) & 440 \\
\hline 64 & $5.98 \mathrm{E}-07$ & 203228_at & PAFAH1B3 & Platelet activating factor acetylhydrolase $1 \mathrm{~b}$ catalytic subunit 3 & 5050 \\
\hline 65 & $6.58 \mathrm{E}-05$ & 33322_i_at & SFN & Stratifin & 2810 \\
\hline 66 & 7.52E-06 & 218976_at & DNAJC12 & Dnaj heat shock protein family (Hsp40) member C12 & 56521 \\
\hline 67 & 9.31E-05 & 200708_at & GOT2 & Glutamic-oxaloacetic transaminase 2 & 2806 \\
\hline
\end{tabular}


Table 1 Datasets used in this study.

\begin{tabular}{llllllll}
\hline Data accession no. & End point & Chip set & Year & Country & Patient no. & Patient no. used & Ref. \\
\hline GSE1456 & OS, RFS & HG-U133A & 2006 & Sweden & 318 & 318 & {$[12]$} \\
GSE11121 & DMFS & HG-U133A & 2008 & Germany & 200 & 200 & {$[14]$} \\
GSE2990 & RFS, DMFS & HG-U133A & 2005 & Canada & 189 & 189 & {$[15]$} \\
GSE15852 & & HG-U133A & 2009 & Malaysia & 86 & 86 & {$[13]$} \\
\hline
\end{tabular}

RFS, relapse-free survival; DMFS, distant metastasis-free survival; DFS, disease-free survival; DSS, disease specific survival; OS, overall survival; DRFS, distace relapse-free survival; MFS, metastasis-free survival. 